\title{
Nonlinear Dirac Neutrino Oscillations
}

\author{
Zhi Hao Quek ${ }^{1, *}$, Wei Khim $\mathrm{Ng}^{1}$, Aik Hui Chan ${ }^{1,}$, and Choo Hiap $\mathrm{Oh}^{1}$, \\ ${ }^{1}$ National University of Singapore \\ Department of Physics \\ 21 Lower Kent Ridge Rd, Singapore 119077
}

\begin{abstract}
Neutrino oscillations are a possible way to probe beyond Standard Model physics. The propagation of Dirac neutrinos in a massive medium is governed by the Dirac equation modified with an effective Hamiltonian that depends on the number density of surrounding matter fields. At the same time, quantum nonlinearities may contribute to neutrino oscillations by further modifying the Dirac equation. A possible nonlinearity is computationally studied using Mathematica at low energies. We find that the presence of a uniform, static background matter distribution may significantly alter the oscillation amplitude and wavelength; the considered nonlinearity may further reduce both oscillation amplitude and wavelength. In addition, the presence of matter allows the effects of the nonlinearity to be more readily observed for the chosen background densities and neutrino energy.
\end{abstract}

\section{Introduction}

Neutrino oscillations are largely regarded as quantum phenomena arising from the mixing between mass and flavour eigenstates. In the presence of surrounding matter, neutrino oscillations would be affected by charged and neutral current interactions.

At the same time, neutrino phenomena present interesting avenues for probing nonstandard physics, such as quantum nonlinearities. These nonlinearities may be crafted via information-theoretic considerations, and function as effective theories; such nonlinearities may manifest as modifications to the effective equations of motion; alternatively, they may occur at the level of the Lagrangian.

In this paper, we consider both matter effects and quantum nonlinearities using a computational model.

\section{Neutrino Oscillations Theory}

Consider the following Lagrangian:

$$
\mathscr{L}=\mathscr{L}_{0}+\mathscr{L}_{\text {Matter }}+\mathscr{L}_{\mathrm{NL}}
$$

where $\mathscr{L}_{0}=\bar{v}\left(i \gamma^{\mu} \partial_{\mu}-m\right) v$ is the usual free Dirac Lagrangian for neutral, spin- $\frac{1}{2}$ particles. $\mathscr{L}_{\text {Matter }}$ and $\mathscr{L}_{\mathrm{NL}}$ represent the matter interaction and nonlinearity terms respectively.

\footnotetext{
*e-mail: zhihao.quek16@sps.nus.edu.sg
} 


\subsection{Matter Interaction}

In standard matter, neutrinos can interact with background matter via charged and neutral current interactions:

$$
\mathscr{L}_{\text {Matter }}=-\sqrt{2} G_{F} \bar{v} \gamma^{\mu}\left[J_{\mu}^{C C} N^{C C}+J_{\mu}^{N C} N^{N C}\right] P_{L} v
$$

In the flavour basis, and for standard matter (whereby electron number density dominates over muon and tau number densities), $N^{C C}=\operatorname{diag}(1,0,0)$ while $N^{N C}=I_{3 \times 3}$. Assuming that the background matter is static and unpolarized, that is $J_{\mu} \propto \rho(x) \delta_{\mu 0}$, we obtain the following equations of motion [1]:

$$
\begin{gathered}
\left(i \gamma^{\mu} \partial_{\mu}-V_{\mathrm{int}} \gamma^{0}\right) v_{L}-m v_{R}=0 \\
\left(i \gamma^{\mu} \partial_{\mu}\right) v_{R}-m v_{L}=0
\end{gathered}
$$

Here, $V_{\text {int }}=-2 G_{F} \sum_{f}\left(T_{3_{f}}-2 Q_{f} \sin ^{2} \theta_{W}\right) \rho_{f} N^{N C}+2 \rho_{e} N^{C C}$, where $f$ represents different fermionic matter fields, $T$ and $Q$ are their isospin, charge and $\theta_{W}$ is the Weinberg angle, and $\rho_{f}$ are the number densities of the various fermionic fields.

We further adopt the chiral representation for the $\gamma$ matrices: $\gamma^{0}=\left(\begin{array}{ll}0 & I \\ I & 0\end{array}\right)$ and $\gamma^{i}=\left(\begin{array}{cc}0 & \sigma^{i} \\ -\sigma^{i} & 0\end{array}\right)$.

The right-handed field in Equation 3 may be eliminated, as per references [2] and [3], to obtain a $2^{\text {nd }}$ order equation of motion, which, for energy eigenstates, has the following conserved quantity:

$$
N \equiv v_{L}^{\dagger}(-i \overleftrightarrow{\nabla}-\vec{\sigma} A) v_{L}
$$

Subsequently, we use the above conserved quantity to normalize our probability distribution; this gives us the following expression for (electron) neutrino survival probability:

$$
P_{e \rightarrow e}=\frac{1}{N} v_{e L}^{\dagger}(-i \overleftrightarrow{\nabla}-\vec{\sigma} A) v_{e L}
$$

\subsection{Quantum Nonlinearities}

As per reference [4] we now proceed to introduce nonlinearities at the level of the Lagrangian. Here, we consider the following nonlinearity:

$$
\mathcal{L}_{N L}=\frac{\left(i \bar{v} \gamma^{K} \partial_{\kappa} v-i\left(\partial_{\kappa} \bar{v}\right) \gamma^{\kappa} v\right)^{2}}{4 A_{\mu} \bar{v} \gamma^{\mu} v}
$$

Here, $A_{\mu}=\left(\frac{1}{A}, 0,0,0\right)$ is a constant timelike vector that results in Lorentz violation and $A$ controls the strength of the nonlinearity. The four-dimensional integral of such a nonlinearity is posited to be an information measure; concomitantly, this nonlinearity satisfies the following information-theoretic axioms:

Firstly, the nonlinearity is homogeneous under re-scaling of $v$, that is $\mathcal{L}_{N L}(\lambda \bar{v}, \lambda v)=$ $\lambda^{2} \mathcal{L}_{N L}$

Secondly, the nonlinearity (and consequently the associated information measure) decrease in value as $v(t, \vec{x})$ tends to a constant value. This is guaranteed by the derivative term in the numerator of the nonlinearity, and corresponds to the intuition that spatial information of a system is lost when the state vector of the system tends to a constant (in the spatial representation).

Thirdly, the theory is local and thus only depends on $v, \bar{v}$ and their derivatives at a point.

Fourthly, the information measure must be positive for arbitrary $v$. 
Finally, the information measure is minimal when the nonlinearity is extremized to obtain the equations of motion.

From the above nonlinearity, we obtain the following modification to the equations of motion:

$$
\begin{aligned}
\partial_{\mu} \frac{\partial \mathcal{L}_{N L}}{\partial(\partial \bar{v})}-\frac{\partial \mathcal{L}_{N L}}{\partial \bar{v}} & =i \gamma^{\mu} v \partial_{\mu}\left[\frac{\left(i \bar{v} \gamma^{\kappa} \partial_{\kappa} v-i\left(\partial_{\kappa} \bar{v}\right) \gamma^{\kappa} v\right)}{2 A_{\sigma} \bar{v} \gamma^{\sigma} v}\right]-A_{\mu} \gamma^{\mu} v\left[\frac{\left(\bar{v} \gamma^{\kappa} \partial_{\kappa} v-\left(\partial_{\kappa} \bar{v}\right) \gamma^{\kappa} v\right)}{2 A_{\sigma} \bar{v} \gamma^{\sigma} v}\right]^{2} \\
& \approx i \gamma^{\mu} v \partial_{\mu}\left[\frac{m \bar{v} v}{A_{\sigma} \bar{v} \gamma^{\sigma} v}\right]+A_{\mu} \gamma^{\mu} v\left[\frac{m \bar{v} v}{A_{\sigma} \bar{v} \gamma^{\sigma} v}\right]^{2} \\
& \approx A_{\mu} \gamma^{\mu} v\left[\frac{m \bar{v} v}{A_{\sigma} \bar{v} \gamma^{\sigma} v}\right]^{2}
\end{aligned}
$$

The first approximation arises from a perturbative calculation using the $0^{\text {th }}$ order Dirac equation. This allows us to sidestep additional boundary conditions and by preventing the equation of motion from becoming a $2^{\text {nd }}$ order PDE. The second approximation uses the fact that the derivatives of the plane-wave solution to the $0^{\text {th }}$ order Dirac equation are null.

\section{Methodology}

Equations 3 and 7 combined give the effective propagation equation for Dirac neutrinos in matter. This EOM is studied numerically in Mathematica via a $4^{\text {th }}$ order Runge Kutta method. The neutrinos are assumed to propagate along 1 dimension only, and are further assumed to be in a negative spin eigenstate, that is, $\sigma^{x} v=-v$. Furthermore, the neutrinos are assumed to be in an energy eigenstate, such that $v(t, \vec{x}) \propto e^{-i E t}$. Finally, the neutrinos are chosen to be initially in the electron flavor eigenstate.

\section{Results}

For ease of discussion, the results are presented in the following manner: matter interaction only, nonlinearity only, and finally both matter and nonlinearity.

\subsection{Matter Interaction Only}

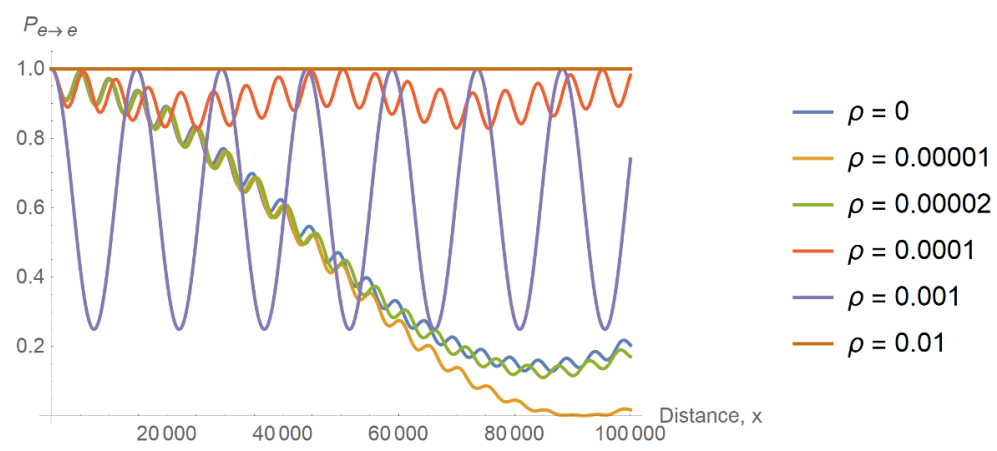

Figure 1. Plot of electron neutrino survival probability, $P_{e \rightarrow e}$, as a function of distance, $x$, for different background densities. 
Here, standard matter is assumed (comprised by protons, neutrons and electrons with equal number densities). In general, the electron neutrino survival probability depends strongly on the background number densities, with high number densities resulting an almost complete suppression of the oscillation phenomena.

\subsection{Nonlinearity Only}

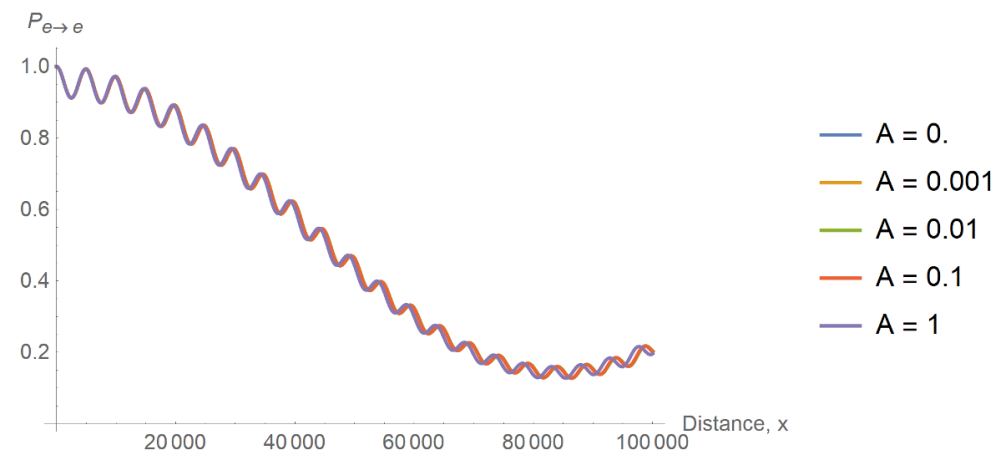

Figure 2. Plot of electron neutrino survival probability, $P_{e \rightarrow e}$, as a function of distance, $x$, for various nonlinearity strengths (different values of $A$ ). A larger $A$ value indicates a stronger nonlinearity.

For the chosen range of nonlinearity strengths, we observe that the nonlinearity term does not significantly affect the oscillation amplitude; even with the largest nonlinearity of $A=1$, we see only a slight shift in the oscillation wavelength with no significant change in amplitude.

\subsection{Both Matter Interaction and Nonlinearity}

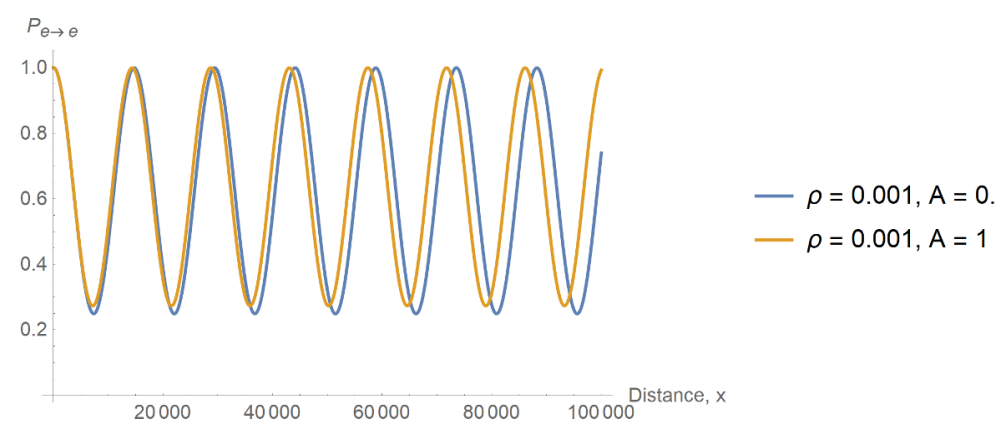

Figure 3. Plot of electron neutrino survival probability, $P_{e \rightarrow e}$, as a function of distance, $x$, for various nonlinearity strengths (different values of $A$ ), for nonzero background matter number density. A larger $A$ value indicates a stronger nonlinearity.

With a nonzero background matter density, we see a much stronger effect with the introduction of the nonlinearity. In particular, we note a noticeable reduction in both wavelength and amplitude. This is in contrast with the much smaller change in oscillation amplitude shown in nonlinearity only case, i.e. Figure 2. 


\section{Conclusion and Future Works}

We implement a modified equation of motion for Dirac particles via quantum nonlinearity to the case of neutrino oscillations in matter. Through our computation results, we see that it is in principle possible to use the phenomena of neutrino oscillations to probe quantum nonlinearities, wherein a sufficiently strong nonlinearity might manifest in a modifications to the survival probability (as a function of distance).

Further studies may explore how the oscillation probability varies as a function of energy. This may prove insightful in determining both the optimal energy for experimental probes of quantum nonlinearities and for placing upper-bounds on nonlinearity values by experiments with fixed detector distance.

\section{Acknowledgements}

The authors would like to thank organizing committee of the Southeast Asian Astronomy Network (SEAAN) Meeting 2019 for a wonderful conference and members of the NUS High Energy Physics group for meaningful discussions.

\section{References}

[1] T. K. Kuo and J. Pantaleone, Rev. Mod. Phys. 61:4, (1989)

[2] Z. Y. Law, A. H. Chan, and C. H. Oh, Int. J. Mod. Phys. A 24, 3483-3492 (2009)

[3] H. T. He, Z. Y. Law, A. H. Chan, and C. H. Oh, in Proc. Conf. Honour Murray GellMann's 80th Birthd. Quantum Mech. Elem. Part. Quantum Cosmol. Complex, (2011)

[4] W. K. Ng and R. R. Parwani, Mod. Phys. Lett. A 26:9, 681-689 (2011) 